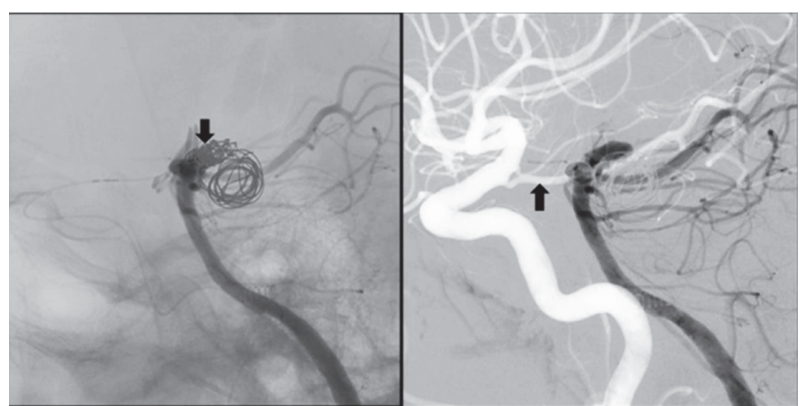

Abstract E-113 Figure 170 yom experienced a basilar artery bifurcation aneurysm recurrence initially after clipping under circulatory arrest and again following basilar to left PCA flow diversion. Dual vessel cerebral angiography (right ICA and left vertebral) reveal a right posterior communicator (up arrow) to the right PCA that was used to access and coil the recurrent basilar aneurysm (down arrow)

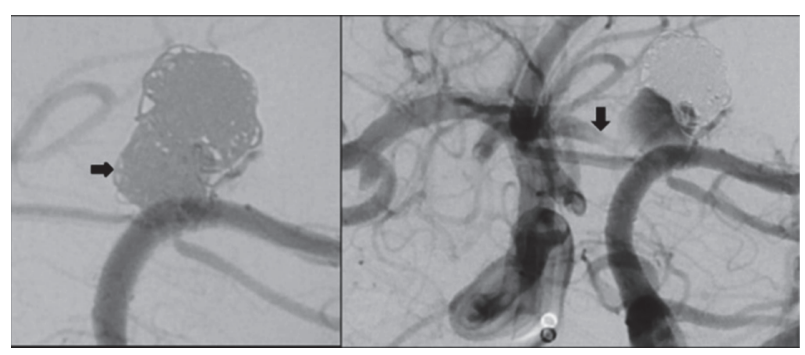

Abstract E-113 Figure 257 yom experienced a basilar artery bifurcation aneurysm recurrence following basilar to right PCA flow diversion. Dual vessel cerebral angiography (left ICA and left vertebral) reveal a left posterior communicator (down arrow) to the left PCA that was used to access and coil the recurrent basilar aneurysm (left arrow) down to the flow diverter

Methods Case series of recurrent aneurysms that were previously clipped and then flow diverted through which access to the recurrence was gained with a microcatheter through the Circle of Willis and then the aneurysm retreated with coiling (figures 1 and 2).

Results Dual injection angiography was used to identify communicating arteries to confirm aneurysm access from adjacent circulation. Once the communication was confirmed, a microcatheter was used to access and coil aneurysm recurrence.

Conclusions Flow diversion for aneurysm recurrence after surgical clipping is a safe alternative to further surgery. Although the Circle of Willis may increase the risk of aneurysm recurrence on flow diverted segments, it provides a valuable conduit through which direct endovascular access to the recurrent aneurysm can be gained for future treatments. Dual injection angiography is required to confirm the presence of these connections before aneurysm access is attempted.

Disclosures J. Steele: None. T. Payner: None. C. Kulwin: None. D. Sahlein: 2; C; Medtronic.

\section{E-114 COILING OF RECURRENT ANEURYSMS FROM THE CONTRALATERAL CIRCULATION FOLLOWING FLOW DIVERSION: TECHNICAL ASPECTS}

1J Steele*, ${ }^{2} \mathrm{~J}$ Scott, ${ }^{2} \mathrm{~A}$ Denardo, ${ }^{2} \mathrm{~K}$ Amuluru, ${ }^{2} \mathrm{D}$ Sahlein. ${ }^{1}$ Michigan Head and Spine Institute, Southfield, MI; ${ }^{2}$ Goodman Campbell Brain and Spine, Carmel, IN
Introduction One concern for aneurysm remnants following flow diversion are the limited retreatment options since the aneurysm sac cannot be directly cannulated through the stent pores. This argument is often used by groups using laser cut stents, where the aneurysm can be re-accessed through the stent, despite their dramatically diminished efficacy and similar risk profile. There are options for retreatment of remnant aneurysms after flow diversion besides placement of more flow diverters, which risks occluding parent vessel branches and increased patient morbidity. The authors discuss the technical aspects of accessing previously flow diverted aneurysms at the base of the brain from the contralateral circulation by using the Circle of Willis.

Methods Dual arterial access recommended, although single artery access possible depending on the anatomy. Dual injection angiography, both hand and power injection, is used to confirm angiographic relationships. Ipsilateral balloon catheter

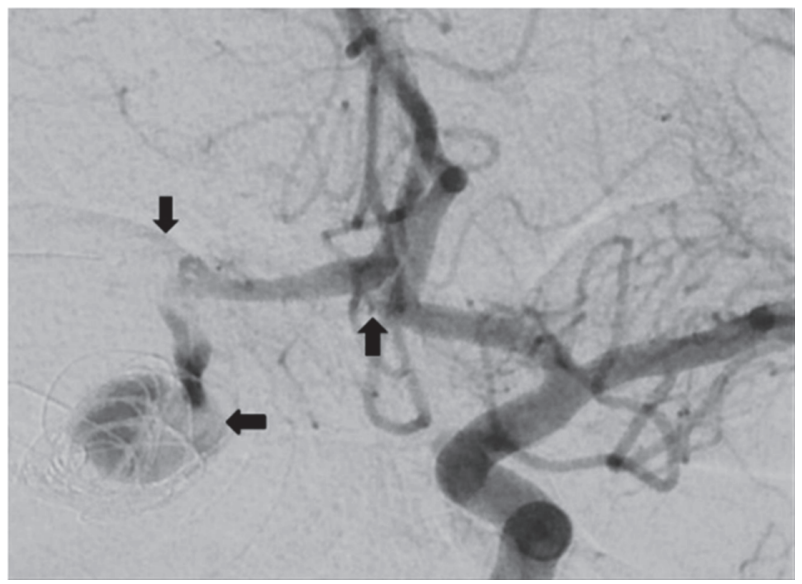

Abstract E-114 Figure 1 Dual vessel access (ipsilateral balloon, down arrow) reveals the acomm (up arrow) through which access to the recurrent pcomm aneurysm (left arrow) was gained.

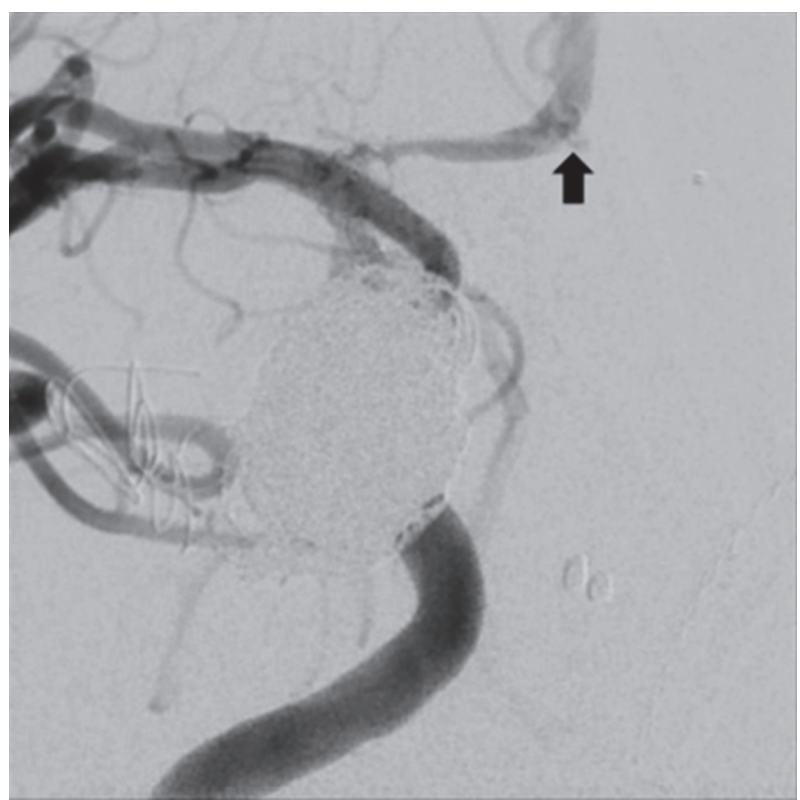

Abstract E-114 Figure 2 Post-treatment through the acomm (up arrow). 
inflation inside the previously placed stent is used to confirm contralateral communication (figure 1).

Results Coil embolization from the contralateral circulation with ipsilateral angiography results in occlusion of the recurrence (figure 2).

Conclusions The Circle of Willis may increase the risk of aneurysm recurrence on flow diverted segments. That being said, it provides a valuable path through which future access to treated aneurysms can be gained. Aneurysm coiling from the contralateral circulation is possible after flow diversion.

Disclosures J. Steele: None. J. Scott: None. A. Denardo: 2; C; Cerenovus. K. Amuluru: None. D. Sahlein: 2; C; Medtronic.

\section{E-115 IDENTIFYING COGNITIVE DYSFUNCTION AFTER ANEURYSMAL SUBARACHNOID HEMORRHAGE}

N Hall ${ }^{*}$, R Chitale, M Fusco, M Froehler. Cerebrovascular Neurosurgery, Vanderbilt University Medical Center, Nashville, TN

\subsection{6/neurintsurg-2021-SNIS.210}

Background Aneurysmal subarachnoid hemorrhage (aSAH) is an often devastating condition, that can result in death or significant neurologic disability. Among the survivors with an otherwise good motor recovery after aSAH, more than half will have cognitive impairment. Implementation of a cognitive assessment strategy after aSAH has not been reported in the literature, despite guideline recommendations to perform cognitive assessment on all stroke patients. Cognitive assessment after aSAH will not only lead to increased recognition of cognitive dysfunction within this patient population, but may also support additional interventions and cognitive therapy to help patients regain function. The aim of this study is to improve cerebrovascular nurse and physician awareness of the significance of the clinical problem and use of objective cognitive assessments for the aSAH patient population.

Methods An educational intervention was developed to bring awareness to the significance of the clinical problem and an understanding of current evidence-based recommendations for cognitive dysfunction screening after aSAH. This was implemented in the Cerebrovascular Clinic associated with a large academic medical center with nurses and physicians caring for the aSAH patient population. Before providing the educational session, the clinicians completed a pretest questionnaire to assess baseline knowledge of cognitive dysfunction after aSAH. The clinicians then participated in a 20-minute peerled education session and completed a posttest questionnaire. A paired samples t-test was used to determine whether there was a statistically significant mean difference between clinician knowledge of cognitive dysfunction in the aSAH population and recommendations for screening by performance on pretest at baseline compared to posttest after the education session.

Results A total of 9 cerebrovascular physicians and nurses completed the pretest, educational session, and posttest. Before the education session, $33 \%$ of participants reported familiarity with specific evidence-based recommendations for cognitive dysfunction screening after aSAH, compared with 100\% after the education session. Clinician average score increased from $71.8 \%$ to $87.8 \%$ after the educational intervention, which is a statistically significant improvement $(p=0.005)$. Seven of the participants had improved scores, and none of the participants had a lower score on posttest than pretest.
Conclusion A brief, peer-led education session on cognitive dysfunction after aSAH improves knowledge of the problem and current recommendations in the literature for cognitive dysfunction after aSAH. Ongoing research is necessary to understand whether the education session and observed positive knowledge change translate to a change in practice and compliance with evidence-based recommendations for cognitive dysfunction screening after aSAH.

Disclosures N. Hall: None. R. Chitale: None. M. Fusco: None. M. Froehler: None.

\section{E-116 NOVEL PERCUTANEOUS ACCESS TO A CONVENTIONALLY INACCESSIBLE CAROTID-CAVERNOUS FISTULA}

${ }^{1} \mathrm{~N}$ Kohler*, ${ }^{1} \mathrm{M}$ Toledo, ${ }^{2} \mathrm{~J}$ Pennington, ${ }^{3} \mathrm{~J}$ Torres-Pacheco. ${ }^{1}$ Department of Neurosurgery, Sacred Heart Hospital, Pensacola, FL; ${ }^{2}$ Department of Otolaryngology, Sacred Heart Hospital, Pensacola, FL; ${ }^{3}$ Sacred Heart Hospital, Pensacola, FL

\subsection{6/neurintsurg-2021-SNIS.211}

Treatment strategies for carotid cavernous fistulas depend on access to the venous outflow of the fistula. The most commonly used route is through the inferior petrosal sinus. Other alternatives are percutaneous access to the superior ophthalmic vein, endovascular transvenous access through the facial vein or infraorbital direct puncture of the cavernous sinus. Transarterial embolization is a less commonly used, but viable alternative. Only one report has described endoscope assisted trans-sphenoidal puncture of the cavernous sinus for direct embolization. ${ }^{1}$ We present the case of a 78 -year-old female who presented with right eye ecchymosis for about 2 months. The initial diagnostic angiogram showed feeders from both external carotid arteries to bilateral cavernous sinus, right greater than left, and associated retrograde venous drainage from the right cavernous sinus into the sphenoparietal sinus and superficial sylvian veins. Initial approach to the fistula was attempted through bilateral inferior and superior petrosal sinuses which failed to demonstrate access to the right cavernous sinus. Further, there was no access to the cavernous sinus through the external venous system. Therefore, we decided to perform an endonasal trans-sphenoidal puncture of the right cavernous sinus in a neurosurgical hybrid operating room with the assistance of Stealth (Medtronic) and Xper guide (Phillips) intra-operative navigation guidance systems. With ENT surgical assistance using rigid endoscopy, stereotactic percutaneous access was obtained of the right cavernous sinus. The right cavernous sinus was then embolized through the trans-nasal route with additional localization and protection utilizing a Scepter XC balloon. Once complete embolization of the right cavernous sinus was achieved, the left cavernous sinus was coiled through the left inferior petrosal sinus. No complications were encountered throughout the entire treatment process and the patient's ecchymosis completely resolved.

\section{REFERENCE}

1. Tang C-L, Liao C-H, Chen W-H, Shen S-C, Lee C-H, Lee H-T, Tsuei Y-S. Endoscope-assisted transsphenoidal puncture of the cavernous sinus for embolization of carotid-cavernous fistula in a neurosurgical hybrid operating suite. Journal of Neurosurgery 2017;127:327-331. doi:10.3171/2016.5.jns16493

Disclosures N. Kohler: None. M. Toledo: None. J. Pennington: None. J. Torres-Pacheco: None. 